\title{
Relaxation effects in the liquid-glass transition range
}

\author{
S. ETIENNE
}

Lab. GEMPPM, UA CNRS 341, INSA Bat 502, 69621 Villeurbanne Cedex, France

\section{Su m mary}

The relaxation effects and the kinetics of the liquid-glass transition are controlled by the molecular mobility with a characteristic time $\tau_{\mathrm{mol}}$. In order to calculate $\tau_{\mathrm{mol}}$, we present a molecular theory based on microscopic concepts of i) quasi-point defects and ii) atomic correlated motions in disordered matter. This model is compared to other theories presented in the literature. The same physical parameters are used to describe the dynamic mechanical behavior and the thermal properties. Some applications are presented, and the spatial microfluctuations of cohesion and enthalpy are shown to be a key point in the dynamic response of disordered matter.

\section{Introduction}

As a glass former is cooled down, molecular mobility decreases and the structure becomes frozen in at a temperature $\mathrm{Tg}$ as indicated by dynamic and calorimetric measurements.

Relaxation effects deal with the time dependent response to a given stimulus. This stimulus can be a field mechanical or electrical stress, leading to the respective responses strain and electrical current (or polarization). Besides the mechanical and electrical relaxations we have structural relaxation observed while applying a thermal history, for example a temperature step. In that case the response is the structural relaxation function $\varphi(t)=\frac{X(t)-X_{\infty}}{X(0)-X_{\infty}}$, where $X$ is a physical property (e.g. the specific volume or enthalpy, the dynamic mechanical modulus, the complex electrical conductivity...). The function $\varphi(t)$ is non-linear and vary with the property $X$.

The relaxation effects are connected to the molecular mobility described by a characteristic time $\tau_{\mathrm{mol}}$ which is the mean time needed by a structural unit to translate over a distance similar to its size through self-diffusion mechanism. This molecular mobility is the key to the time dependent responses and the kinetic transition from the liquid to the glassy state.

\section{A new approach of the molecular mobility in the disordered matter.}

Classical interpretations of the molecular mobility and of the liquid-glass transition are reported in the literature. The work of Cohen and Turnbull (1) is based on the free volume concept and gives a relation for $\tau_{\mathrm{mol}}$ as:

$$
\tau_{\mathrm{mol}}=\tau_{\mathrm{o}} \exp \left(-\frac{\mathrm{v} 1}{\mathrm{vf}}\right)
$$

$v 1$ is the volume of one structural unit, $\mathrm{vf}=\mathrm{v} 1 \Delta \alpha$ (T-T0) is the free volume per structural unit which redistributes without change of energy, $\Delta \alpha$ is the difference of expansivity coefficient between the liquid and the glassy states. $\tau_{0}$ is about $10^{-13} \mathrm{~s}$. The prominent problem of this theory is that no temperature dependence is predicted in the isostructural state at $\mathrm{T}<\mathrm{Tg}$ when the free volume is frozen in. Its applicability is thus 
restricted to the liquid state at high temperatures. The theory of Adam and Gibbs (2) is based on the notion of cooperative motions leading to thermally activated events in a sub-region containing $z^{*}$ structural units. The mean frequency of such rearrangements is proportional to $\exp \left(-\frac{\mathrm{z}^{*} \Delta \mu}{\mathrm{kT}}\right) . \Delta \mu$ is the activation barrier for the jump of one structural unit. $z^{*}$ has a minimum value equal to 2 . Then, $\tau_{\mathrm{mol}}$ can be written:

$$
\begin{aligned}
& \tau_{\mathrm{mol}}=\tau_{0} \exp \frac{\mathrm{U}^{*}}{\mathrm{kT} \ln (\mathrm{T} / \mathrm{T} 2)} \\
& \text { with: } \mathrm{U}^{*}=\frac{\mathrm{k} \ln 2 \Delta \mu}{\Delta \mathrm{Cp}}
\end{aligned}
$$

$\Delta \mathrm{Cp}$ is the increment of heat capacity at $\mathrm{Tg}$ and $\mathrm{T} 2$ is the temperature at which the configurational entropy of the liquid state would be zero.

Several observations are to be mentioned. The assumption of the configurational entropy uniformly distributed among the structural units is implicit.

Both formulations would be equivalent if the specific volume and entropy where order parameters, which is not the case. It is well known (3) that liquid glass transition occuring at $\mathrm{Tg}$ is related to a Prigogine-Defay ratio greater than unity, which implies a multi-parameter description.

When the microstructure is frozen in $(T<T g)$, the dynamic properties of the glassy state remain temperature dependent. Only the entropy frustration theory yields a temperature dependence of $\tau_{\mathrm{mol}}$ with an apparent activation energy equal to $\mathrm{U}^{*} / \ln (\mathrm{Tg} / \mathrm{T} 2)$.

Recently, mode coupling theories (4) where presented to describe the slowing down of molecular motions as the matter is cooled from the liquid state. According to this theory, the mean time $\tau_{\mathrm{mol}}$ for the displacement of a structural unit by coherent vibrations and cage effects is found to obey the equation:

$$
\tau_{\mathrm{mol}}=\tau_{0}\left[\frac{\mathrm{T}-\mathrm{Tc}}{\mathrm{Tc}}\right]-\gamma
$$

The viscosity $\eta$ is derived from $\tau_{\mathrm{mol}}$ :

$$
\eta=\eta_{0}\left[\frac{T-T c}{T c}\right]^{-\gamma}
$$

The critical temperature $\mathrm{Tc}$ lies at about $\mathrm{Tg}+50^{\circ} \mathrm{C}$. Experimental observations using quasielastio neutron scattering seem to confirm this slowing down at $\mathrm{Tc}$ where the density autocorrelation function can be fitted by stretched exponential laws.

Some aspects remain however unclear: i) autocorrelation functions are only related to the first peak of the dynamic structure factor ii) short times $\left(10^{-13}\right.$ to $\left.10^{-8} \mathrm{~s}\right)$ are considered: what happens at longer times? iii) thermally activated diffusion processes are not clearly considered.

Thus, taking into account the limitations of the theories presented above, we present an approach based on thermal activation and self-diffusion assisted molecular dynamics. This model, first proposed by Perez et al $(5,6,7)$, is based on the concepts of quasi-point defects (qpd) and of correlated atomic motions. The microfluctuations of density lead to microfluctuations of cohesion. Less cohesive domains are called defects (figure 1). In the metastable state $(\mathrm{T}>\mathrm{Tg})$, the concentration $\mathrm{C}$ of these defects is controlled by the reaction:

$$
2 \mathrm{~d}_{0} \rightleftarrows \mathrm{d}_{+}+\mathrm{d}_{-}(\Delta \mathrm{Hf}, \Delta \mathrm{Sf}, \Delta \mathrm{v})
$$

$\Delta \mathrm{Hf}, \Delta \mathrm{Sf}, \Delta \mathrm{v}$ are increment of enthalpy, entropy and volume associated to the formation of a defect. In equilibrium, the concentration is given by the Boltzman statistics:

$$
C=\frac{1}{1+\exp \left(\frac{\Delta H f}{k T}\right) \exp \left(-\frac{\Delta S f}{k}\right)}
$$


Below $\mathrm{Tg}, \mathrm{C}$ has not its equilibrium value and we have the structural relaxation the kinetics of which is controlled by the structural relaxation time $\tau_{\mathrm{sr}}$ following the relation:

$$
\frac{\mathrm{dC}(\mathrm{t})}{\mathrm{dt}}=\frac{\mathrm{C}(\mathrm{t})-\mathrm{C}_{\infty}}{\tau_{\mathrm{sr}}}
$$

The second concept introduced in our theory is the correlation between atomic motion in the disordered matter. Let us consider a thermally activated jump of a structural unit with a mean time $\tau 1$. As the jump has occured, structural units in the surroundings rearrange leading to a time dependent relaxation time $\tau(t)=\tau 1\left(t_{1 / t_{0}}\right)^{1-b}>\tau 1$. These correlated motions extend over a time $\tau_{\max }$. Writing that $\tau(\mathrm{t})$ is equal to $\tau_{\max }$ for $\mathrm{t}=\tau_{\max }$ we have $(6,7)$ :

$$
\tau_{\max }=\left[\frac{\tau 1}{t_{0}}\right] 1 / \mathrm{b} t_{0}, \text { which is identified to } \tau_{\mathrm{mol}}
$$

$\tau 1$ is the elemental motion characteristic time, to is a scaling factor time and $0<b<1$ is correlation factor $(b=1$ : no correlation effects and $\tau(t)$ remains equal to $\tau 1$, while these effects are maximum when $b$ goes to zero). A similar expression was derived from the coupling model developed by Ngai and coworkers $(8,9,10)$, but we have attached the following physical meaning to the factors $\tau 1$ and $b: \tau 1$ is identified to the characteristic time of the secondary relaxation, and we assume that $b$ is a function of the microstructural state trough $\mathrm{C}$.

The third ingredient of this molecular theory is the spatial distribution of the characteristic time $\tau_{\mathrm{mol}}$. This distribution is considered by taking into account a spatial distribution of $\mathrm{C}$ (i.e. of the correlation factor $\mathrm{b}$ ), in agreement with the conclusion of Fischer (11) who claimes the existence of microfluctuations of density at different scales by means of quasi-elastic light scattering. Thus, eqs with $b$ like eq 4 have to be distributed.

With $\tau_{\mathrm{mol}}$, we can calculate the time dependent response. Let us consider the disordered matter submitted to a low stress field (mechanical relaxation in the linear regime): as the stress is applied, rearrangements occur in the most disordered sites (i.e. the defects) resulting in the nucleation of shear microdomains (characteristic time $\tau 1$ ). Due to correlation effects, these domains expand. The non-elastic strain is recoverable because of the elastic energy of the Somigliana lines boarding these domains. If the stress is applied for a long time, neighbouring domains percolate, the energy of the lines disappears and we have a non-recoverable strain component. After calculation of the compliance $J(t)$ and its Fourier transform $J(j \omega)$, the complex dynamic modulus is deduced:

$$
\begin{aligned}
& J(t)=\frac{1}{G \infty}\left[1+A\left(1-\exp -\left(\frac{t}{\tau_{r}}\right) b\right)+A\left(\frac{t}{\tau_{r}}\right)\right] \\
& G(j \omega)=\frac{1}{J(j \omega)}=G_{\infty}\left[\frac{1}{1+\left(j \omega \tau_{\alpha}\right)^{-b}+q\left(j \omega \tau_{\alpha}\right)^{-1}}\right]
\end{aligned}
$$

$A$ is a quantity proportional to $C, G_{\infty}$ is the modulus at infinite frequency and $q$ is a function of $A, G$ and $b$.

It can be shown that the macroscopic mechanical relaxation times $\tau_{r}$ and $\tau_{\alpha}$ are not very different from $\tau_{\mathrm{mol}}(6,7)$.

In the case of polymeric materials, correlation effects increase as the local shear strain increases, and we have to consider entropic elasticity (12), thus a modified expression is derived for $G(j \omega)$ :

$$
G(j \omega)=\frac{1}{J(j \omega)}=G_{r}+\left(G_{\infty}-G r\right)\left[\frac{1}{1+\left(j \omega \tau_{\alpha}\right)^{-b}+q\left(j \omega \tau_{\alpha}\right)^{-b}}\right]
$$

$\mathrm{Gr}$ is the relaxed (entropic modulus) and $0<\mathrm{b}<\mathrm{b}^{\prime}<1$.

Let us now consider the structural relaxation process. For sake of simplicity, we ignore the spatial distribution of $C$. This relaxation is described by the characteristic time $\tau_{\mathrm{sr}}$. 

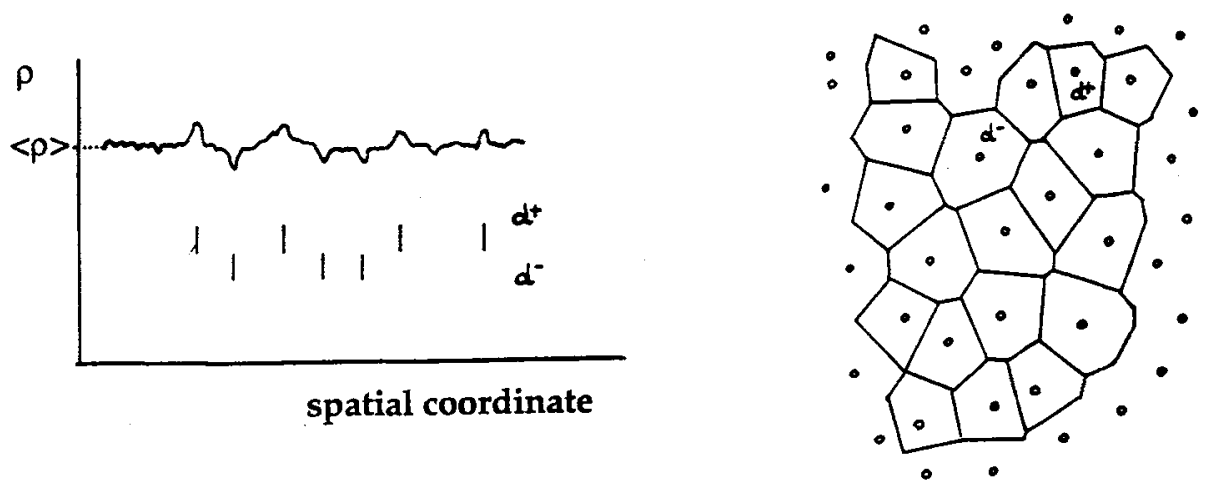

Figure 1: schematic representation of disordered matter. Microdomains where the density is particularly low (or high) are called $d-$ (or $d+$ ) quasi-point defects. The defects can be observed in the Wigner Seitz cells representation.

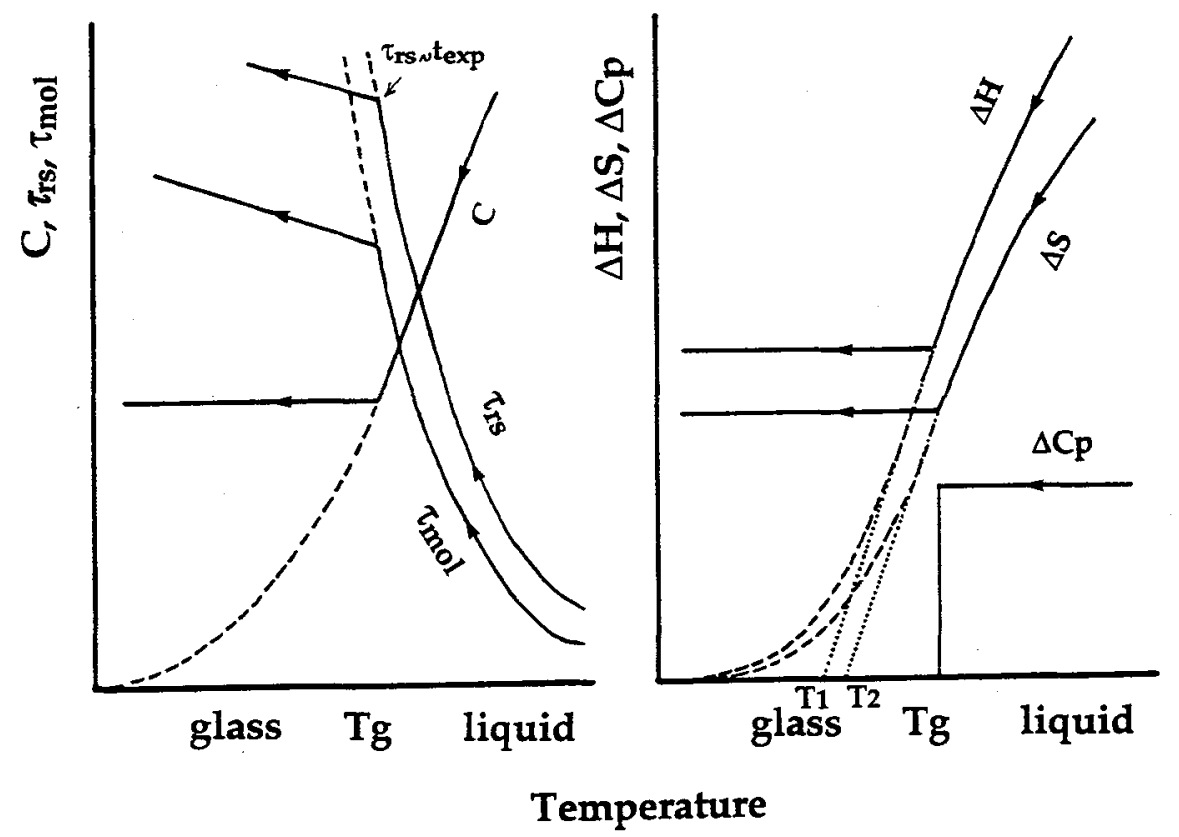

Figure 2: schematic evolution of disordered matter properties on cooling from the liquid (metastable equilibrium state) to the glassy state. The glass transition at $\mathrm{Tg}$ corresponds to the freezing when the structural relaxation time $\tau_{\mathrm{rs}}$ is comparable to the experimental time $t$ exp. Naturally, there is no sharp transition at $\mathrm{Tg}$ but rather a gradual slow down. $\mathrm{T} 1$ and $\mathrm{T} 2(=\mathrm{T} 0)$ are temperatures at which extrapolation from the liquid state leads $\Delta \mathrm{H}$ and $\Delta \mathrm{S}$ (or free volume) to vanish, respectively (see text). Dashed lines are predictions of the qpd model for infinitely low cooling rate. 
This time is controlled by the self diffusion process. If $D$ is the diffusion coefficient and $\lambda$ and $l$ are the size of a structural unit and the mean distance between defects respectively, we can write:

$$
\tau_{\mathrm{sr}} \sim \frac{1^{2}}{\mathrm{D}} \quad \mathrm{D} \sim \frac{\lambda^{2}}{\tau_{\mathrm{mol}}} \quad \frac{1}{\lambda} \sim \mathrm{C}^{-1 / 3}
$$

Combining these quantities we obtain the time dependent $\tau_{\text {sr }}(t)$ during the structural relaxation:

$$
\tau_{s r}(t)=\left[\frac{\tau 1}{t_{0} 1-b(t)}\right]^{1 / b(t)} \quad C(t)^{-2 / 3}>\tau_{\operatorname{mol}}(t)
$$

Combining eqs 3 and 7 we obtain the well known non linear aspect of structural relaxation function.

To sum up, our approach of the molecular mobility is consistent with the different features of the relaxation effects in the liquid-glass transition range:

-the high apparent activation energy Uapp in isostructural conditions is explained. From eq 4 Uapp is that of $\tau_{\mathrm{mol}}$

$$
\text { Uapp }=\frac{\mathrm{U} 1}{\mathrm{~b}}>\mathrm{U} 1
$$

-above $\mathrm{Tg}$, the WLF law is consistent with a temperature dependent correlation factor. -the multiorder parameter nature is in agreement with: i) a Prigogine-Defay ratio $R>1$ and ii) memory effects well described first by Kovacs (13).

-a stretched exponential component is present in the relaxation function -non linear aspects of the structural relaxation

Furthermore, the parameters introduced to describe the dynamic behavior are connected to thermal properties. If we neglect the component of phonons, the heat capacity $\mathrm{Cp}$ is given by the variation of the number of defects against temperature $T$. The excess of thermal properties with respect to the crystal is:

$$
\begin{aligned}
\Delta \mathrm{Cp} & =\Delta \mathrm{Hf} \mathrm{Na} \frac{\mathrm{dC}}{\mathrm{dT}} \\
\Delta \mathrm{S} & =\Delta \mathrm{Sf} \mathrm{NaC}+\text { mixing entropy of } \mathrm{NaC} \text { defects among Na sites } \\
\Delta \mathrm{H} & =\Delta \mathrm{Hf} \mathrm{NaC}
\end{aligned}
$$

Within this frame, the liquid glass transition temperature is the temperature $\mathrm{Tg}$ at which the structural relaxation time is similar to the experimental observation time. This transition is kinetic in nature, and we see from eqs 1,2 and 8 that the temperature at which $\Delta \mathrm{H}, \Delta \mathrm{S}$ and $\Delta \mathrm{v}$ should vanish in the metastable equilibrium state is $0 \mathrm{~K}$. Thus Kauzman paradox appears to have no consistency (figure 2).

\section{Manifestations of relaxations, discussion}

Experimental observation on relaxation effects near $\mathrm{Tg}$ are analysed and compared with theoretical predictions.

\section{Mechanical relaxation}

A quantitative description of the mechanical relaxation near the liquid-glass transition range can be derived from eqs 5 and 6 . Numerous results related to polymeric and non-polymeric materials in agreement with theoretical predictions of qpd model are presented elsewhere (see for example references 14,15 and 16). We focus in this paper our attention on the case of flow (i.e. the low frequency limit of the dynamic behavior) of non-polymeric materials and its dependence on microstructure. In order to study the effect of the microstructure, materials with increasing ratio Rws between weak and strong interatomic cohesive forces were considered. Figure 3 presents results for silica, soda lime glass and glucose. From the diagram it is observed that the fragility $(17,18)$ of the liquid is an increasing function of Rws. In order to clarify this point in 


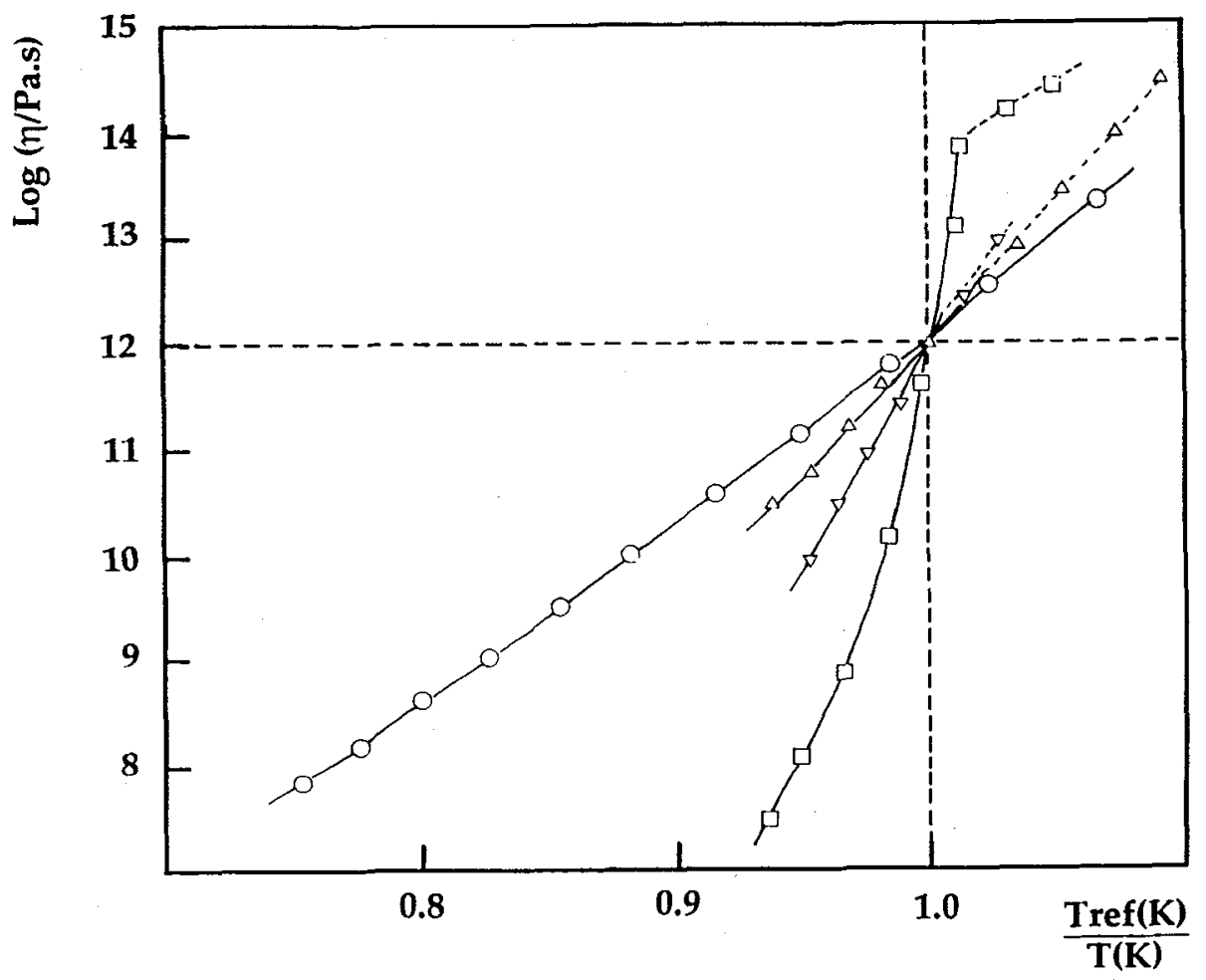

Figure 3: viscosity $\eta$ plotted against the reduced reciprocal temperature. Tref is the temperature at which $\eta$ is equal to $10^{12} \mathrm{~Pa}$.s. Tref is equal to $1493 \mathrm{~K}, 843 \mathrm{~K}$ and $305 \mathrm{~K}$ for silica $(0)$, calcium-sodium silicate $(\Delta)$ and glucose $(\square)$ respectively. This representation shows the transition from strong (e.g. silica) to fragile (e.g. glucose) liquid. The change of slope in the high viscosity range (dashed lines) corresponds to the transition from the metastable equilibrium state to the non equilibrium or glassy state. Results plotted for an oxynitride $(\nabla$ Tref $=1077 \mathrm{~K})$ suggest that this material is a rather fragile liquid.

Table I

$\begin{array}{llllll}\text { Material } & \begin{array}{l}\mathrm{Tg} \\ (\mathrm{K})\end{array} & \begin{array}{l}\Delta \mathrm{Cp} \text { at } \mathrm{Tg} \\ (\mathrm{J} / \mathrm{mol} / \mathrm{K})\end{array} & \begin{array}{l}\Delta \mathrm{Hf} \\ (\mathrm{kJ} / \mathrm{mol})\end{array} & \begin{array}{l}\Delta \mathrm{Sf} \\ (\mathrm{J} / \mathrm{mol} / \mathrm{K})\end{array} & \mathrm{b} \\ \text { glucose } & 308 & 166 & 23 & 77 & 0.17 \\ \text { silica } & 1495 & 1.44 & 52 & 3.6 & 0.43\end{array}$


the frame of qpd model, the correlation factor was deduced from Cole Cole plots. Measurements give $b=0.43,0.37$ and 0.17 for silica, binary $\mathrm{Na}_{2} \mathrm{O}, 3 \mathrm{SiO}_{2}$ and glucose, respectively. Thus, correlation effects appear to increase with Rws and fragility. The conclusion that is deduced is mainly: in a strong liquid, i.e. silica, the motion of a structural unit implies breaking a strong interatomic bond ( $\Delta \mathrm{Hf}$ is high). The qpd is localised on this bond ( $\Delta \mathrm{Hf}$ is low). On the contrary in a fragile liquid, e. g. glucose, only weak interatomic bonds are involved in the motion of a molecule ( $\Delta \mathrm{Hf}$ is low). The qpd extend over several molecules and $\Delta \mathrm{Sf}$ is high. From thermal analysis data and using eqs 5 , the enthalpy and entropy of defects formation can be calculated. The results displayed in table $I$ are in agreement with the theoretical prediction quoted above.

\section{Structural relaxation}

In the liquid-glass transition range, the relaxation time $\tau_{\mathrm{rs}}$ is of the same order as the experimental time. If the material is not at equilibrium, time dependent properties can be observed. As quoted before, the structural relaxation deals with the evolution of the microstructure against time. If we characterize this microstructure by the concentration $\mathrm{C}$, it is evident from eqs 3 and 5 that thermal and mechanical spectrometry experiments are convenient to study this relaxation process. The first example we discuss is the interpretation of DSC results obtained after physical aging below Tg. Such a study was carried out by Vigier et al (19) in the case of Poly(Ethylene Terephtalate). Using the qpd model with a distribution introduced on the parameter $b$, a good agreement is found between experimental results and theoretical predictions: in particular i) the sub- $\mathrm{Tg}$ peak of $\Delta \mathrm{Cp}$ exhibited by specimen aged at low temperature and ii) the shift of the endotherm peak with aging time. The second example is the determination of glass transition temperature by means of mechanical spectrometry at constant heating rate. This procedure was used for metallic glasses (20) and nonmetallic glasses (21). It was shown that in the high frequency limit $\left(\omega \tau_{\mathrm{mol}} \gg 1\right)$ the term

$\mathrm{d} \ln (\tan \varphi) / \mathrm{dT}$ varies as $\mathrm{Cp}$, but with an enhanced sensitivity (21). This is particular interesting in the case of matcrials exhibiting low $\Delta \mathrm{Cp}$ increment at $\mathrm{Tg}$.

Besides thermal history (e.g. physical aging or quenching), high rate plastic deformation is another way to modify the microstructural state. At high stress levels and high strain rates, the plastic deformation of disordered matter (i.e. Somigliana lines motions) implies creation of defects because the self-diffusion assisted process described above is not efficient enough. Illustration is given on figure 4 where simulated curves relative to Poly(Styrene) are plotted. Figure 5 shows the effect of plastic deformation on the dynamic shear modulus (i.e. of molecular mobility) of Poly(Methyl Metacrylate). Specimens of PMMA were deformed at room temperature by plane strain compression mode between parallel dies at different levels (B,C,D and E), then the stress was released. Figure 5 shows the behavior presented by the specimen deformed up to level D. Measurements were carried out on heating at a rate $60 \mathrm{~K} /$ hour. If we compare the deformed and the undeformed specimen (case A), it can be seen that the plastic deformation has induced a high molecular mobility in the material as the shift from $370 \mathrm{~K}$ towards $320 \mathrm{~K}$ of the loss factor rising indicates. Above $340 \mathrm{~K}$ the structural relaxation manifests and near $380 \mathrm{~K}$, both curves merge, i.e. the effect of deformation on the microstructure is erased. In order to compare effects of thermal history and plastic deformation, we have drawn on the figure results obtained for a sample heated above $\mathrm{Tg}$ at $443 \mathrm{~K}$ for 120 seconds and then quenched in cold water. Quenching appears to be much less efficient to modify the microstructure. Let us now discuss these observations. It is well known that quenching from $\mathrm{T}>\mathrm{Tg}$ decreases the density $\rho$. Actually we measured at room temperature $\rho=1.887 \mathrm{~g} / \mathrm{cm}^{3}$ and $\rho=1.883 \mathrm{~g} / \mathrm{cm}^{3}$ for the as received and the quenched specimen, respectively. As shown on figure 5 , the plastic deformation has an opposite effect on $\rho$ while molecular mobility is drastically increased (this increase of density by the deformation mode used is due to i) the compression component of the strain and ii) the alignement of macromolecular chains). 


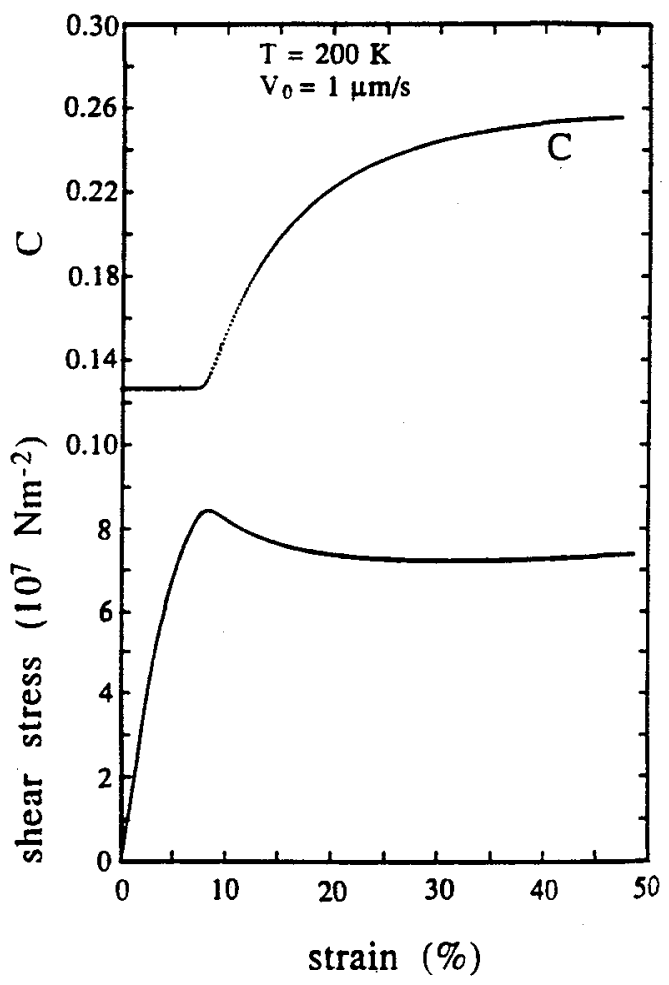

Figure 4: Simulation from the apd model of defect creation by high rate plastic deformation of Poly(Styrene) at low temperature. The yield stress corresponds to the onset of microstructural defects creation. From reference (7). 


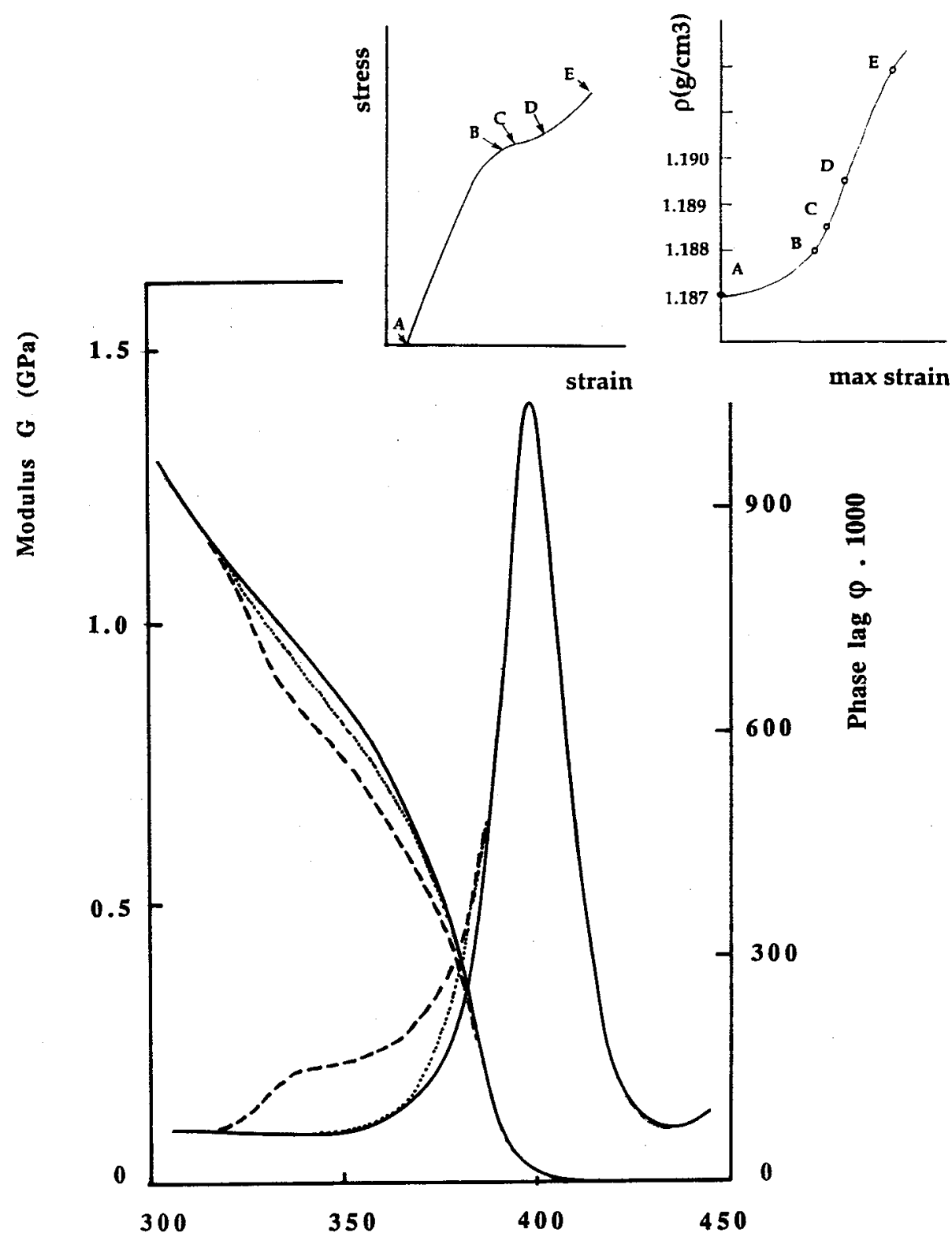

Temperature (K)

Figure 5: Effect of plastic deformation and quenching on dynamic properties of PMMA. Isochronal $(0.1 \mathrm{~Hz})$ mechanical spectrometry measurements were carried out on heating at a rate $60 \mathrm{~K} / \mathrm{h}$. The stress/strain curve and density (measured with a gradient column) measured at room temperature after releasing the stress are shown in the inset. (full line: aged specimen, dashed line: deformed state and dotted line: quenched state). 


\section{Conclusion}

Intensive work is carried out for modelling the liquid-glass transition and related relaxational behavior.

We have presented a model based on concepts of quasi-point defects (i.e. microfluctuations of density) and correlated atomic motions. This model appears to be in good agreement with the experimental features.

Compared results on the effect of deformation and thermal history show evidence that microfluctuations and not the mean value of specific volume are a prominent factor. Quasi elastic light scattering are in progress to clarify this point. It can be noticed that the spatial distribution of $\tau_{\mathrm{mol}}$ is consistent with the concept of percolation $(23,24)$ with liquid like and solid like microcells in the $\mathrm{Tg}$ region.

\section{References}

[1] COHEN, M.H. and TURNBUlL, D., J. Chem. Phys., 31 (1959) 1164

[2] ADAM, G. and GIBBS, J.H., J. Chem. Phys., 43 (1965) 139

[3] DAVIES, R.O. and JONES, G.O., Adv. Phys. 2 (1954) 370

[4] LEUTHEUSSER, E., Phys. Rev. A 29 (1984) 2765

BENGTZELIUS, U., GOETZE, U. and SJOELANDER A., J. Phys. C17 (1984) 5915

[5] PEREZ, J., J. Phys. 46 (1985) 427

[6] PEREZ, J., CAVAILlE, J.Y., ETIENNE, S. and JOURDAN, C., Rev. Phys. Appl., 23 (1988) 125

[7] PEREZ, J., Physique et mécanique des polymères amorphes, Lavoisier Paris (1992)

[8] NGAI, K.L., Comments Solid State Phys. 9 (1979) 127

[9] NGAI, K.L., comments Solid State Phys. 9 (1980) 141

[10] NGAI, K.L., RENDELL, R.W., RAJAGOPAL, K. and TEITLER, S., Ann. NY Acad. Sci. 484 (1986) 150

[11] FISCHER, E.W., in "Basic Features of the Glassy State", p172, J. Colmenero and A. Alegria Eds., World Scientific (1990)

[12] FERRY, J.D., Viscoelastic Properties of Polymers, 3rd edition, Wiley, New York (1980)

[13] KOVACS, A.J., Fortschr. Hochpolym. Forsch. 3 (1963) 370

[14] CAVAILLE, J.Y., PEREZ, J. and JOHARI, G.P., J. non cryst. solids, 131-133 (1991) 935

[15] SEKKAT, A. and ETIENNE, S., to be published

[16] DAVID, L. and ETIENNE, S., to be published

[17] ANGELL, C.A. and SICHINA, W., ann. NY Acad. Sci., 27 (1976) 53

[18] ANGELL, C.A, J. non cryst. solids, 131-133 (1991) 13

[19] VIGIER, G., J. TATIBOUET, J. and J. PEREZ, Proceedings 3th Workshop on noncrystalline solids, Seville (1991)

[20] SINNING, H.R. and HAESSNER, F., Scripta Metall. 20 (1986) 1541

[21] PEREZ, J., ETIENNE, S. and TATIBOUET, J., Phys. Stat. Sol. (a) 121 (1990) 129

[23] CHEN, H.S., J. non cryst. solids, 46 (1981) 289

[24] COHEN, M.H. and GREST, G.S., Phys. Rev. B, 20 (1979) 1077 